Canadian University Music Review

Canadian University Music Review

Revue de musique des universités canadiennes

\title{
De l'histoire écrite à l'histoire orale : la danse guerrière de La Todolella
}

\section{Ramón Pelinski}

Volume 12, numéro 1, 1992

URI : https://id.erudit.org/iderudit/1014212ar

DOI : https://doi.org/10.7202/1014212ar

Aller au sommaire du numéro

Éditeur(s)

Canadian University Music Society / Société de musique des universités canadiennes

ISSN

0710-0353 (imprimé)

2291-2436 (numérique)

Découvrir la revue

Citer cet article

Pelinski, R. (1992). De l'histoire écrite à l'histoire orale : la danse guerrière de La Todolella. Canadian University Music Review / Revue de musique des universités canadiennes, 12(1), 75-94. https://doi.org/10.7202/1014212ar

All Rights Reserved (C Canadian University Music Society / Société de musique des universités canadiennes, 1991
Ce document est protégé par la loi sur le droit d'auteur. L’utilisation des services d'Érudit (y compris la reproduction) est assujettie à sa politique d'utilisation que vous pouvez consulter en ligne.

https://apropos.erudit.org/fr/usagers/politique-dutilisation/ 


\title{
DE L'HISTOIRE ÉCRITE À L'HISTOIRE ORALE : LA DANSE GUERRIÈRE DE LA TODOLELLA
}

\author{
Ramón Pelinski
}

\section{Introduction}

En 1990-91, pendant une année sabbatique ${ }^{1}$, j'ai eu la chance de faire de longs séjours au Maestrazgo (province de Castellón, Espagne) et de dialoguer souvent avec les habitants de La Todolella ; dans les conversations, l'une des questions suscitées avec le plus de fréquence portait sur l'ancienneté de leur Danse guerrière.

À cause de sa relative complexité chorégraphique, de son pouvoir d'identification culturelle et de sa prétendue antiquité, la Danse guerrière de La Todolella est l'une des expressions « choréo-musicales » les plus appréciées dans le Maestrazgo.

Pour arriver à mieux comprendre le problème de ses origines historiques, j'ai entrepris de rédiger les notes qui suivent. Ces notes ne sont pas exhaustives, mais j'espère qu'elles pourront contribuer à éclairer la question depuis une nouvelle perspective.

Je me propose donc :

a) d'examiner de manière critique quelques documents sur la Danse guerrière, documents provenant aussi bien de l'histoire écrite que de la tradition orale;

b) de réaliser une brève analyse stylistique du texte musical de la Danse, pour confronter ensuite les résultats de cette analyse avec les documents mentionnés.

Il s'agit ici de montrer comment les perspectives synchronique et diachronique peuvent être utilisées pour élargir notre compréhension du fait culturel. En

${ }^{1}$ Je remercie le Ministère de l'éducation d'Espagne dont l'aide m'a permis de passer neuf mois, en tant que chercheur adjoint, à la U.E.I. de musicologie de l'Institució Milà i Fontanals (Barcelone) du Consejo Superior de Investigaciones Cientificas. J'exprime aussi ma reconnaissance à la Conselleria de Cultura, Educació i Ciencia de la Generalitat Valenciana, qui a subventionné une partie de mon travail de terrain au Maestrazgo, sans oublier l'Université de Montréal, qui m'a octroyé cette année sabbatique. Le présent article est la version modifiée d'un texte publié dans Anuario Musical, C.S.I.C. (Musicologie), Barcelone, 1991. 
d'autres termes, mon but est d'éclaircir, à partir du cas particulier de la Danse guerrière de La Todolella, l'interaction des perspectives historique et anthropologique.

La réalisation de cet objectif pré-suppose qu'il faut postuler l'unité de la musicologie comme « une discipline dans laquelle la diversité des traditions musicales peut être étudiée dans une variété de perspectives interreliées » (Widdes $1989: 1$ ). S'il est certain que « toute compréhension du présent qui ignore son interaction avec le passé sera sérieusement incomplète » (Widdes, ibid.), l'affirmation inverse est aussi vraie : la compréhension du passé est impossible sans la conscience de son interaction avec le présent.

Voici la démarche que je suivrai.

En premier lieu, je ferai une relecture critique des documents de l'histoire écrite, à partir d'un double point de vue : en tant que traces d'une pratique artistique, et en tant que traces d'une représentation idéologique de cette pratique. Dans le premier cas, il s'agit d'examiner une série de documents qui témoignent objectivement de l'existence de la Danse guerrière dans le passé. Dans le second cas, ces documents seront considérés comme des témoignages d'une mentalité selon laquelle le discours sur une expression artistique déterminée est utilisé comme exercice du « pouvoir sur la mémoire future » (Le Goff 1988 : 304). L'histoire écrite d'une pratique artistique peut devenir en elle-même objet d'une histoire, quand la représentation d'une idéologie (conception globale de la société) ou d'une mentalité (structures mentales communes à une catégorie sociale ou à une époque) se comprend comme construction d'une réalité historique (Ibid. : 23).

En deuxième lieu, je comparerai les résultats de cette analyse historiographique à des documents qui proviennent de l'histoire orale. Fondée sur la mémoire des paysans, soumise aux distorsions de l'oubli et aux changements de perspectives des générations, l'histoire orale constitue néanmoins la source principale du savoir collectif. La mémoire collective - en particulier, sa " mise en action » durant une exécution choréo-musicale - est la « zone silencieuse » dans laquelle le «monde oublié du paysan » (de Certeau $1974: 27$ ) récupère et recrée son identité culturelle.

Devant la pénurie de documents écrits, l'histoire orale présente des avantages considérables pour le chercheur. Fondée sur le passé de courte durée, c'est-à-dire un passé dont la profondeur se mesure par la capacité de la mémoire d'un individu ou d'une collectivité (Hobsbawn 1972), l'histoire orale abonde en témoignages d'expériences culturelles immédiates, vécues. Plus encore, l'histoire 
orale est mémoire de « ce qui reste du passé dans l'expérience des groupes, ou ce que les groupes font du passé » (P. Nora 1978).

En ce sens, l'histoire orale est une rencontre avec l'histoire écrite. Loin de s'épuiser dans la représentation d'un passé limité au souvenir de ce qui, effectivement, s'est passé, la mémoire collective des paysans est peuplée des fantômes d'un passé dans lequel mythe et histoire se confondent. Au-delà des expressions conscientes de leur mémoire et de l'apparent changement des choses, les paysans parlent de la Danse guerrière comme d'une pratique « très ancienne "; ils en parlent comme s'ils imaginaient ces « courants internes qui traversent en silence la longue durée de l'histoire » (Detienne $1986: 74)$. « Le mythe réinvente un nouveau niveau de l'histoire : l'histoire lente » (Le Goff $1988: 230$ ), ou l'histoire dans « la limite de l'immobile »(Braudel $1968: 74$ ).

Prétendre prouverl'antiquité de la Danse guerrière au moyen de traits stylistiques, formels ou immanents peut être discutable. En effet, comme l'a observé Josep Martí i Perez dans une communication personnelle sur le présent travail, une argumentation sur l'ancienneté de cette danse devrait prendre en considération non seulement des traits formels, mais aussi des traits culturels comme l'iconographie, la chorégraphie, l'habillement, etc. Plus encore, si cette expression artistique n'est pas un produit figé, mais qu'elle s'inscrit dans une continuité de changements culturels et stylistiques, comment pourrait-on chercher des preuves de ses origines à travers des traits purement stylistiques? Je réponds à cette objection en disant que : à l'effet de déterminer l'ancienneté de la Danse, le manque de sources premières oblige le chercheur à se prévaloir de la réalité aujourd'hui existante (musique, instruments, chorégraphie, vêtements, modes de transmission, etc.) et à examiner ses implications historiques. Toutefois, dans le cas du présent travail, je me limiterai à l'examen de la musique, laissant l'analyse des autres traces à la monographie que je prépare sur la tradition musicale de La Todolella.

\section{Documents historiographiques}

Il n'est pas étrange que, dans une communauté de type rural de tradition fondamentalement orale, la question de l'origine d'une pratique culturelle suscite d'autant plus d'intérêt qu'il y a très peu documents historiques qui en parlent. Ceci semble être le cas de La Todolella, où les rares documents écrits qui mentionnent une activité musicale ne sont aucunement transparents en ce qui concerne la Danse guerrière.

2.1. Le Docteur Josep Eixarch a trouvé dans les livres de comptes de La 
Todolella la première mention d'une activité « choréique » dans ce village ${ }^{2}$ : en 1654 , le village de Zorita prête de petites cloches pour les danses de La Todolella, sans que l'on spécifie de quelles danses il s'agissait (Serra i Fortuño 1988).

2.2. Selon Mossén Milián, il existait dans les archives paroissiales de La Todolella un manuscrit daté de 1758, dont le titre disait : « Yntroduction, Loa y Dichos de San Joaquín para el año 1756 con sus Despedidas. Hablan 8 Danz(antes), y un Galán, y Gracioso "3 (1950: 2) 4 . La Danse guerrière actuelle serait la même que celle à laquelle réfère ce manuscrit.

2.3. Un document inestimable pour l'histoire de la Danse guerrière date de 1929. Je fais allusion à la transcription musicale que le dolçainer ${ }^{5}$ de Cinctorres, José Ripollés Climent ('Piou') a réalisée sous le titre de: Dansas (« Danses ») ou Dansas típicas ( Danses typiques»), selon le post-scriptum. Piou offrit sa transcription au village de La Todolella le 3 juin 1929, avec la dédicace suivante : « Regalo al pueblo de La Todolella. A no dejarlas perder "6. Plus loin, je reviendrai sur ce document avec plus de détails.

2.4. En 1950, dans un article intitulé « La Dança Todolellana. Fué transmitida cuidadosamente de padres a hijos " ${ }^{\prime 7}$ Mossén Milián affirme que La Todolella ...

conserve dans le réservoir de ses coutumes un témoignage remarquable de richesse folklorique pour sa typique et très ancienne Danse. Sans doute s'agit-il d'un rare joyau médiéval, souvenir de ses divertissements poétiques et imprégné de musique, de poésie, de militarisme, de vie pastorale et d'amours populaires, avec lequel le peuple célébrait ses fêtes et réjouissances. Ainsi, la Danse de La Todolella naquit après la Reconquête, se dansant dans les Fêtes patronales, et elle est arrivée intacte jusqu'à nos jours, fait dont il reste une survivance documentaire dans un manuscrit de 1756, conservé dans les archives paroissiales (Homenaje a M. Milián 1987 : 19).

2.5. En 1951, Manuel Palau déclare que « À La Todolella, on interprète une danse très ancienne dans laquelle on simule une bataille » $(1951: 101)$.

${ }^{2}$ Malheureusement, je n'ai pu consulter suffisamment ces livres car leur état précaire l'interdit, jusqu'au jour ils seront restaurés.

${ }_{3}$ « Introduction, Louange et Dits de saint Joachim pour l'an 1756 avec ses strophes finales. Il y a 8 dans(eurs), et un Galant, et un Bouffon.

${ }^{4}$ Ce manuscrit a été publié par G. Puerto Mezquita (1956 : 166-173).

${ }^{5}$ Joueur de dolçaina, hautbois populaire de la région de Valence. (N.d.É.)

6 "Cadeau au village de La Todolella. Ne pas laisser perdre. "

7 « La danse todolellanaise. Elle fut transmise soigneusement de pères en fils ». 
2.6. L'étude la plus importante sur la Danse guerrière se trouve dans l'œuvre Danzas procesionales de Morella y del Maestrazgo ${ }^{8}$ (1956), de Gonzalo Puerto Mezquita. Parlant des danses de Forcall, l'auteur affirme que : « ces danses sont très anciennes. Sans doute, sont-elles nées entre le XIV et le XVI ${ }^{e}$ siècle, soit à la même époque où apparaissent celles des villages voisins : Morella, Zorita et La Todolella. » (1956: 18)

Se référant spécifiquement à la Danse guerrière qui nous occupe, Puerto Mezquita pense que celle-ci « apparaît dans notre folklore, selon les meilleurs témoignages, entre le $\mathrm{XIV}^{\mathrm{e}}$ et le XVI $\mathrm{XV}^{\mathrm{e}}$ siècle » (1956:50). Ces témoignages seraient les suivants :

- Vers la fin du $X V^{e}$ siècle, on voit deux types de danse, qui sont fondamentales : la marche, en mesure de $2 / 4$, et le saut (salto), plus animé, en mesure de $3 / 4$. La Danse guerrière possède des caractéristiques qui proviennent de ces deux types. (1956:51)

- La danse guerrière espagnole n'est pas un dérivé de la danse pyrrhique; au contraire, elle lui est antérieure! Les danses guerrières sont une survivance de l'ancienne civilisation ibérique. Les Touraniens furent ceux qui instituèrent la liturgie chorégraphique; $c$ 'est ainsi que la famille ibérique a introduit les danses religieuses dans la péninsule. (1956: $51-$ 52)

2.7. En 1980, dans une étude intitulée «Las danzas guerreras en Todolella et Peñiscola », le folkloriste Antonio Manuel Carreras Alvar situe la Danse guerrière dans la tradition des peintures rupestres de la zone valencienne et de la culture courtisane « à laquelle avait accès le seigneur féodal du château [de La Todolella] »; s'associant à l'hypothèse de Puerto Mezquita, il déclare :

en fusionnant les études de danse et les études musicales, nous pouvons arriver à la conclusion que la Danse guerrière de La Todolella a surgi dans la première moitié du $\mathrm{XVI}^{\mathrm{e}}$ siècle, ce que sa musique même révèle, bien qu'elle possède des mouvements qui existaient déjà au $X^{e}$ siècle. (1980 : 21)

Je ne crois pas trahir le contenu des témoignages exposés plus haut en les condensant dans les propositions suivantes :

a) En général, on doit chercher l'origine des danses guerrières espagnoles dans les pratiques ibériques, plus anciennes que la danse pyrrhique grecque (Puerto

8 « Danses processionnelles de Morella et du Maestrazgo ». 
Mezquita), ou dans les danses inspirées des peintures rupestres de la région valencienne (Carreras Alvar).

b) En particulier, l'origine de la Danse guerrière est très ancienne (Palau et Puerto Mezquita); elle se situe soit au Moyen-âge d'après la Reconquête (Mossén Milián), soit entre le Moyen-âge et la Renaissance (Puerto Mezquita), soit encore dans le passage du XVe au XVI ${ }^{e}$ siècle (Carreras Alvar).

c) La Danse guerrière, exécutée dans les Fêtes patronales, " est arrivée intacte jusqu'à nos jours » (Mossén Milián).

d) La Danse guerrière pourrait avoir ses origines entre le XIVe et le XVI ${ }^{e}$ siècle, à cause de l'existence de danses de rythme binaire et ternaire au $\mathrm{XV}^{\mathrm{e}}$ siècle (Puerto Mezquita).

e) Il est probable que la Danse guerrière actuelle soit en relation avec les danses auxquelles fait référence le manuscrit de 1756 (Mossén Milián).

Il est pourtant possible de mettre en doute ces propositions si on les confronte aux observations suivantes :

a) Les auteurs mentionnés n'offrent aucun document qui prouve leurs affirmations; celles-ci sont donc reléguées aux limbes des hypothèses non vérifiées.

b) Plus particulièrement, l'affirmation selon laquelle la Danse guerrière « est arrivée intacte jusqu'à nos jours » (Mossén Milián) contredit toutes les évidences que l'on possède sur la dynamique inhérente au processus folklorique, processus qui se manifeste non seulement dans la variabilité de la structure musicale interne, mais aussi dans la conceptualisation et l'exécution de la musique de tradition orale.

c) Si l'existence, au $X^{e}$ siècle, de danses de rythme binaire et ternaire constituait vraiment une preuve de l'origine "renaissante » des danses guerrières, pourquoi ne pas penser aussi que l'Hymne de l'Internationale socialiste et l'Hymne national polonais, respectivement fondés sur des rythmes binaires et ternaires, proviennent de la Renaissance?

d) Il se peut que la Danse guerrière soit liée à la présence de 8 danseurs, comme le signale le manuscrit de 1756, mais rien ne permet actuellement de le prouver.

En dépit de tout cela, nous serions injustes avec les auteurs ci-haut mentionnés si nous nous contentions d'écarter leurs témoignages à cause d'un manque de preuves historiques ou empiriques. Un document faux est aussi un document 
historique, et il peut s'avérer un témoignage précieux de l'époque à laquelle il fut forgé (Le Goff 1988 : 303). En effet, ces témoignages sont importants parce qu'ils s'inscrivent à l'intérieur d'un discours symbolique dont la validité est garantie par le fait qu'ils nous révèlent des aspects de la culture de la région qui transcendent la simple controverse de l'origine historique d'une danse particulière. Il s'agit de témoignages qui, au niveau du discours historiographique, confirment l'existence de certaines valeurs culturelles inhérentes à la vie des paysans du Maestrazgo. Parmi ces valeurs culturelles, signalons les deux suivantes :

- la stabilité et la continuité de la culture, laquelle, malgré la variabilité relative de ses manifestations artistiques, crée une ambiance de sécurité existentielle, grâce à la répétition de rites et pratiques inscrits dans le cycle annuel des saisons, cycle qui fonde le temps circulaire de la liturgie;

- le sentiment d'identité culturelle qui, à un niveau local ou régional, entretient un sentiment de différence vis-à-vis des autres villages de la région. La Todolella possède une « star » : sa Danse guerrière.

Ces deux aspects ont été confirmés dans les entrevues que j'ai réalisées durant cette année passée avec les paysans de La Todolella.

\section{Documents de l'histoire orale}

La Todolella est une communauté paysanne qui, en dépit des nouveautés et des transformations des dernières décennies (mécanisation de la campagne, électrification, téléphone, télévision, etc.), a conservé fidèlement deux de ses expressions musicales les plus caractéristiques : la Santatonada ${ }^{9}$ et, évidemment, la Danse guerrière.

La perte d'autres traditions, telles que le chant des Albadas, les chants de dépiquage et les massejades, est acceptée avec résignation par la génération plus âgée et considérée par les plus jeunes comme un signe de décadence. Mais il vaut la peine de signaler que c'est justement la jeune génération qui consacre une partie de ses énergies et de son temps à la conservation, la restauration et la revitalisation des deux monuments que leur tradition musicale leur a légués.

Selon les habitudes paysannes, la conservation de ces manifestations artistiques se fait par tradition orale : la mémoire passive, qui conserve la compétence de ses expressions culturelles, et la mémoire active, qui se développe dans le processus de l'apprentissage, de l'exécution et de la réception. Dans les deux cas, il y a des

${ }^{9}$ C'est la fête de saint Antoine, célébrée dans le temps du Carnaval. Elle est centrée sur le symbolisme des anciens rites du feu et de la fécondité (Bouché Peris 1982 : 185-229). 
spécialistes de la mémoire, tacitement reconnus par la communauté en tant que tels, dont le rôle est de maintenir vive la mémoire des expressions culturelles et, ce faisant, de contribuer à la cohérence sociale de la communauté. En ce qui a trait à la Danse guerrière, les porteurs de mémoire sont le cap de dansa (« chef de danse »), le dolçainer, l'un des danseurs ou quelque membre de la communauté, particulièrement intéressés à la conservation de la Danse.

Les témoignages que j'ai recueillis sur l'histoire orale de la Danse contiennent deux types d'informations :

- des narrations d'événements reliés à l'histoire de la Danse à partir du XX siècle. Les témoignages qui réfèrent à son histoire immédiate sont particulièrement abondants, étant donné que le passage du folklore au folklorisme, ou de la tradition au traditionalisme (Baumann 1976; Martí i Perez 1990) stimule la mémoire collective et son objectivation en chronologies, conversations, discussions et exécutions publiques;

- des témoignages de la mémoire collective, parfois vagues et confus, qui font appel à la vénérable longévité de la Danse.

Les deux types de témoignages font respectivement référence à des durées différentes. Dans le premier cas, il s'agit d'une histoire orale attentive aux événements du « temps bref, dans la mesure des individus, de la vie quotidienne » (Braudel $1968:$ :65). Tandis que dans le second cas, la sensibilité au passé se révèle dans la notion d'une pratique culturelle inscrite dans une histoire de longue durée (Braudel, ibid. : 70).

Je suis conscient du fait que le chercheur, malgré l'état d'empathie où il se trouve avec la culture observée, ne devrait pas abandonner une attitude critique vis-àvis des paroles de ses collaborateurs-paysans, comme d'ailleurs ceux-ci exercent souvent leur sens critique vis-à-vis des observations du chercheur. Il ne s'agit pas de fétichiser les mots des collaborateurs, ni ceux du chercheur.

Je me limiterai à signaler quelques-uns seulement des témoignages qui illustrent les deux types de durée ci-haut mentionnés.

3.1. D'un côté, selon les témoignages de la tradition orale qui s'orientent vers une histoire de longue durée, les Todollellanais pensent que « la Danse guerrière est très vieille ». Ainsi l'affirme Monsieur Manuel Membrado Llop (81 ans), et ainsi le confirme celui qui fut dolçainer de la Danse entre 1950 et 1980 :

Je ne sais pas d'où est sorti ceci [la Danse guerrière], qui l'aurait amenée ici; je ne sais pas en quelle année elle a été fondée. Les archives ont été brûlées, c'est vrai, mais il devrait y avoir un livre qui dirait d'où venait ceci, de quoi 
ça venait, qui l'a amenée ici, comment elle s'est formée. Cela doit faire beaucoup beaucoup d'années, boh! (Entrevue avec José M. Armengot, 12-10-90.)

Vicent Sorribes, gérant et danseur de la formation actuelle, me disait :

Nous avons seulement une date, qui est 1654. Mais cela ne nous convainct pas. Nous, nous pensons qu'elle est beaucoup plus ancienne. Alors, nous sommes en train de chercher d'où elle vient cette danse. Pourquoi La Todolella et pas un autre village? La relation qui existe entre les danses d'Aragon, les danses du Pays basque, celles du centre de l'Europe, est-ce qu'il s'agit d'un mouvement né ici ou d'un mouvement importé? (Entrevue du 14-9-90.)

Amelia Ferrer, qui fut directrice de danse de la Section féminine de Castellón, et elle-même danseuse de la Danse guerrière, est catégorique : « Cette danse est du $\mathrm{XI}^{\mathrm{e}}$ siècle, selon les données que possédait la Section féminine dans une archive qui a brûlé ». (Entrevue du 13-12-90.)

3.2. D'un autre côté, les narrations de l'histoire orale immédiate apportent des témoignages qui, débordant généreusement la limite de l'histoire écrite, nous informent sur des aspects fondamentaux de la conceptualisation, de la signification sociale et de la structure formelle de la Danse. Ainsi pouvons-nous savoir que :

\subsubsection{La danse guerrière est propriété municipale :}

Nous avons appris la Danse vers 1948 ou 49. Nous sommes allées parler avec le maire de La Todolella qui, à cette époque-là, était Monsieur Miguel de Barrón, pour voir si eux, ils voulaient nous l'enseigner. Mais il y a eu beaucoup de difficultés, parce que c'était une danse d'hommes; ils pensaient que nous allions leur enlever la Danse, et pour cette raison ils ne voulaient pas nous l'enseigner. Et alors, nous leur avons dit : « Nous n'allons pas vous enlever cette Danse. S'il y avait des hommes dans notre groupe, ce seraient eux qui la danseraient; mais ça serait un malheur que cette Danse se perde et que l'Espagne ne la connaisse pas. Nous, nous n'allons pas présenter cette Danse comme étant la nôtre, mais nous la danserons toujours avec le nom de La Todolella, parce que, finalement, cette Danse elle vient de là. » Et alors, finalement, ils ont accepté. (Entrevue avec Amelia Ferrer, 13-12-90.)

3.2.2. La formation de la Danse guerrière est dictée par un principe héréditaire : c'est une tradition confirmée par les danseurs que le rôle de «chef de 
danse », en particulier, est hérité par descendance familiale directe. Ce principe a parfois été appliqué aux autres rôles de la Danse et il s'est même étendu à la danse des Gitanetes («Petits Gitans »)-exécutée par des femmes et rituellement associée à la Danse guerrière -, dont les rôles ont également été assignés à des parents des danseurs.

\subsubsection{Son apprentissage s'effectuait par transmission orale :}

Et alors, les danseurs nous l'enseignèrent. Et nous avons appris la Danse en dansant, parce que eux ne savaient pas l'enseigner. Ils étaient déjà âgés. Ils la dansaient, ôtaient un homme et le remplaçaient par une fille. Bon, ils te laissaient les mains...! Et après, quand elle avait déjà une idée, ils en mettaient une autre. Quand ils nous ont laissés seules, tu ne peux pas te faire une idée, ce jour-là on ne voyait plus que des bâtons. (Entrevue avec Amelia Ferrer, 13-1290.)

À la maisonj'ai un livre de danses qui est écrit par un certain Mezquita. Vous ne l'avez pas vu? Celui qui est là-bas, droit comme un bâton, ça c'est Antonio Martí Ramia. Ce monsieur, c'est celui qui est venu de Zorita. On lui a enseigné notre Danse quand j'étais petit. Il a appris très très vite, et tout par oreille. On lui a enseigné en chantant! (Entrevue avec José M. Armengot, 12-10-90.)

Monsieur Rafael Prats (85 ans) se souvient qu'après le pèlerinage de 1943, la Danse « s'est perdue » pendant quelques années. Elle réapparut vers 1950, sur l'initiative de l'oncle Cuquilla, qui l'enseigna au dolçainer et aux danseurs, en chantant les mélodies de ses diverses parties.

\subsubsection{Les occasions et le contexte de son exécution étaient rituelles :}

À l'ermitage de saint Christophe [San Cristóbal], on a dansé la Danse guerrière quand j'étais petit; parce que, dans ce temps-là, il n'y avait pas une image ni rien du saint. Et alors, ce saint Christophe qu'il y a maintenant làhaut, il a été amené par quelqu'un de Barcelone, quelqu'un qu'on appelait Cinctorranet parce que ses parents, je ne sais pas, ont vécu là, dans le mas de Cinctorrà. Il est venu ici et a amené ce saint, et il a dit que nous devions faire une bonne fête. Alors on a fait le pèlerinage traditionnel à pied, on a présenté «L'Intermède du Bouffon » [Entremés del Gracioso], et on a dansé les Gitanetes et la Danse guerrière. Quand la statue a quitté le village les gens chantaient :

Noble saint Christophe tu quittes ce village 
mais ne nous oublie pas

quand tu seras là-haut

sur la montagne.

Quand on danse la Danse dans la procession de la Saint-Bartholomé, on part de l'église et on arrive jusqu'à la Place : on danse toutes les danses et on retourne jusqu'à la porte de l'église en ramenant l'image de saint Bartholomé. Quand je disais : « Allons-y, allons à la Place », à telle heure on dansait la danse. Quand arrivait le prêtre avec son chapeau, le maire et les autres autorités, ils se mettaient tous au balcon de la mairie, tous ceux-là, et alors les danseurs s'approchaient du $\mathrm{dao}^{10}$ pour faire les danses. Eh oui! L'alguacil observait s'il y avait quelque chose qui manquait ou si les gens s'avançaient trop, et, enfin, c'était joli tout ça! L'alguacil avait un bérêt sur la tête et il disait : « Allez-y par en arrière ». Et le maire disait à l'alguacil : « Dites-leur qu'ils peuvent déjà commencer ». (Entrevue avec José M. Armengot, 1210-90.)

3.2.5. L'exécution rituelle de la Danse à l'occasion des Fête patronales n'empêchait pas qu'elle pût aussi être représentée lors de circonstances spéciales. Selon le témoignage de Manuel Membrado Llop, dit « Arabogas » (81 ans) :

La première fois que j'ai vu la Danse,j'avais six ans. C'était en 1916. Quand il y a eu la grippe espagnole en 1918, on a descendu le saint de l'ermitage au village. Des affaires de curé! Alors, quand ils ont remonté le saint le 3 mai, il y a eu beaucoup de monde ici. Avec la grippe, plus de 60 personnes sont mortes dans le village. (Entrevue du 4-9-90.)

À cette époque, on dansait la Danse parfois quand venaient les fêtes ou quand il y avait une occasion. Une fois, il y en a un qui est venu de la France, il était de cette ferme là-haut et on l'appelait Masset. Il était parti en France quand il était jeune. Et il a dit au « chef de danse » : « Allez, vous devez me danser la Danse. Je vais vous tuer un agneau et vous devez venir ». Ensuite, il voulait aussi qu'on s'en aille en France, mais nous ne sommes pas allés. (Entrevue avec José M. Armengot, 12-10-90.)

3.2.6. Étant donné les changements de générations, la pratique de la Danse ne pouvait être toujours soumise à un régime d'exécution annuel strict. À cette discontinuité fait allusion le témoignage suivant :

Après qu'ils ont fait cette fête de saint Christophe, dans laquelle on a repris la Danse, la Danse s'est arrêtée pendant plusieurs années; après, on l'a

${ }^{10}$ Carré en pierre dessiné sur la Grand' Place du village et destiné à l'exécution de la Danse guerrière. 
abandonnée à nouveau. Puis, on l'a reprise quand m'a fait jouer pour la première fois un homme de ce village qu' on appelait oncle Cuquilla. Et, bien sûr, il y a eu tant d'époques, on l'a abandonnée et ensuite on l'a renouvelée; évidemment, il n'y avait pas de continuité. À cette époque-là, on faisait le même nombre de danses qu'aujourd'hui. On dit qu'anciennement, il y avait deux danses de plus et qu'elles se sont perdues. (Entrevue avec José M. Armengot 12-10-90.)

3.2.7. Cette discontinuité peut expliquer les changements esthétiques et structurels qui contredisent le mythe de la transmission « intacte jusqu'à nos jours » de la Danse guerrière. Ainsi, Monsieur Rafael Prats se souvient que, peu avant la guerre civile, l'oncle Cuquilla et, après lui, le danseur Terencio disaient qu'on pouvait altérer l'ordre de chacune des danses si ce qu'on voulait c'était obtenir une plus grande plasticité dans l'exécution. Après la guerre civile, Monsieur Prats était présent à une répétition exécutée à la mairie, au cours de laquelle l'oncle Cuquilla signala qu'anciennement la danse avait comporté jusqu'à 19 pièces.

3.2.8. La Danse guerrière accomplit une fonction importante dans le développement du sentiment d'identité locale :

La Danse est importante pour le village. Ici, à La Todolella, nous avons trois choses : la Danse, l'ermitage de saint Christophe et le château, qui sont trois choses assez renommées déjà. Les mélodies de la Danse ne se retrouvent nulle part ailleurs. Je ne les ai entendues nulle part, jamais. Parfois, je suis allé jouer dans d'autres villages où il y a une petite danse, et comme il n'y en a qu'une seule, donc 'tata rata', tu l'apprends tout de suite. Mais attention! Ici à La Todolella, il y a douze danses [12 pièces] et ça a son mérite. Il y a deux ou trois ans, je suis allé à Zorita jouer pour leur danse; bah!, une petite danse, 'tralala', et c'est fini. 'Tralala', c'est fait, il n'y avait rien de plus. Toujours la même. Il y a toutes sortes de danses mais comme la nôtre, il n'y en a pas hostie!, non, non. Ça a son grand mérite. (Entrevue avec José M. Armengot, 12-10-90.)

Alors, nous nous sommes demandés comment une chose qui est de La Todolella et qui a été dansée par mon grand-père, mon père et tous mes ancêtres, pourquoi ce devait être notre génération qui briserait la chaîne. Nous serions coupables si une chose aussi vieille - que l'on doit encore découvrir - devait se perdre. Les gens dansent la Danse parce qu'elle est de leur village, parce que leurs parents l'ont dansée, leurs grands-pères, leurs oncles. C'est clair. Un village aussi petit que La Todolella qui soit si grand 
dans le folklore! C'est ça qui nous motive, c'est ça qui nous rend orgueilleux d'être Todolellanais. La Danse est comme un ambassadeur de La Todolella. À Castellón, y'a pas beaucoup de personnes qui savent où se trouve La Todolella, mais tout le monde connaît sa Danse. (Entrevue avec Vicent Sorribes, 14-9-90.)

3.2.9. L'histoire orale se fait à partir de « lieux de la mémoire collective » (P. Nora 1984), comme le dao de la Grand' Place ou la montagne qui abrite l'ermitage de saint Christophe :

Sur le sol de la Place, il y avait le dao. C'est dommage qu'on l'a caché : on l'a recouvert de ciment. J'aurais laissé ce carré en pierre où, anciennement, on dansait les Jotas ${ }^{11}$ et la Danse. Tous dansaient sur ce carré et voyez donc maintenant : on l'a caché, oui, je ne sais pas comment ils ont fait ça. Bah, on fait ça pour les rues, d'accord, mais au moins, il aurait fallu respecter ce carré de danse sur la Place, là où on faisait toutes les choses. Oh! la la! Oui, ces choses anciennes étaient très belles. Maintenant non. Maintenant, tout est moderne, pas plus. (Entrevue avec José M. Armengot, 12-10-90.)

Je voulais transcrire tous ces témoignages in extenso pour montrer quelle mine de renseignements peut nous transmettre l'histoire orale et dans quelle mesure ces informations peuvent contribuer à l'esquisse d'une histoire écrite devant l'absence de documents écrits.

\section{Le Texte musical}

Pour tenter d'élucider l'origine de la Danse guerrière, je présenterai maintenant une brève analyse formelle du texte musical de la Danse. Des 14 parties qui la constituent, soit :

1. Les entraetes

2. L'entrada del ballet

3. El villano per baix ${ }^{12}$

4. El villano per dalt

5. El ballet nou

6. El tintaro

7. El villano de pau i bastó

8. La samarreta

${ }^{11}$ Danses d'origine aragonaise (N.d.É.).

12 Villano : littéralement, "roturier ». Danse espagnole des $\mathrm{XVI}^{\mathrm{e}}$ et $\mathrm{XVII}^{\mathrm{e}}$ siècles qui essayait d'imiter les danses rustiques. 
9. El serenge

10. El retaule (Le retable)

11. La Filus bella

12. El Mambrú (Malbrough)

13. El torn (Le tour)

14. Les despedides (Les strophes finales),

il y a trois mélodies qui sont respectivement identiques à trois autres pièces. Les entraetes $\left(\mathrm{n}^{\circ} 1\right)$ sont identiques à Les despedides $\left(\mathrm{n}^{\circ} 14\right)$, El serenge $\left(\mathrm{n}^{\circ} 9\right)$ à La Filus bella $\left(\mathrm{n}^{\circ} 11\right)$, et le Villano per dalt $\left(\mathrm{n}^{\circ} 4\right)$ au Villano de pau $i$ bastó $\left(\mathrm{n}^{\circ} 7\right)$. Malgré l'impression d'homogénéité que lui donnent son accompagnement instrumental (dolçaina et tabalet ${ }^{13}$ ) et son acceptation traditionnelle comme une totalité « choréo-musicale » typique de La Todolella, le style musical de la Danse guerrière est hétérogène.

4.1. À en juger par la structure modèle de la mélodie de L'entrada del ballet $\left(n^{\circ} 2\right)$ et du Serenge $\left(n^{\circ} 9\right)$, ces deux pièces pourraient se situer avant la fin du $\mathrm{XVII}^{e}$ siècle, soit avant la généralisation de l'emploi de la tonalité majeuremineure.

4.2. La formule harmonique(I-IV-I-I-V-I) et la conséquente ligne mélodique du Villano per baix (no 3) remontent à la tradition renaissante du Villano espagnol et se retrouvent avec une certaine fréquence dans la musique folklorique espagnole (Olmos 1951 ; Querol Gavaldá 1956 : 25 ; García Matos 1959-61 : 290).

Il est curieux que de noter que dans l'inscription de la Danse offerte par Piou à la mairie de La Todolella, le Tintaro $\left(\mathrm{n}^{\circ} 6\right)$ commence aussi avec une mélodie qui correspond au modèle du Villano espagnol renaissant. Cette mélodie n'est pas exécutée dans l'actuelle version de la Danse.

4.3. Les mélodies avec rythmes aksak (en turc : «boiteux »), aussi appelés « rythmes amalgamés » ou « bichrones irréguliers », sont le Ballet nou $\left(n^{\circ} 5\right)$, le Tintaro $\left(n^{\circ} 6\right)$, la Samarreta $\left(n^{\circ} 8\right)$ et le Serenge $\left(n^{\circ} 9\right)$. Bien que le mètre de Les entraetes $\left(\mathrm{n}^{\circ} 1\right)$ et de $L^{\prime}$ entrada del ballet $\left(\mathrm{n}^{\circ} 2\right)$ donne, à la première écoute, une impression de symétrie, il est possible - comme me l'a signalé Vicent Torrent $i$ Centelles lors d'une conversation personnelle et comme j'ai moi-même pu le vérifier par expérience - que ces pièces contiennent des vestiges du rythme aksak. En effet, l'accent agogique (subtile élongation temporelle) que, tout particulièrement, le tabalet pose sur les deux premières unités de la mesure m'a 13 Tambour. 




Figure 1

8. La samarreta
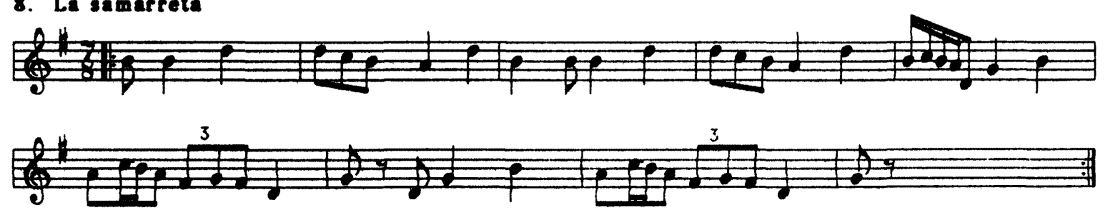

Figure 2

amené à interpréter l'articulation régulière de $2+2+2$ (ou de $3+3$ ) comme intentionnellement orientée vers un rythme de $3+2$ (ou de $4+3$ ). Un sonogramme réalisé sur un analyseur de parole avec le logiciel VIS/PC V3 186, développé par Robert Marchand au Laboratoire de linguistique de l'Université de Montréal, a confirmé cette hypothèse.

L'intérêt de ces rythmes réside dans le fait qu'ils s'inscrivent dans une tradition commune à des pays circonvoisins du bassin de la Méditerranée (Brailoiu 1973 : 303-340), tradition dont l'existence peut être retracée jusqu'à la musique grecque ancienne (Georgiades 1949). Il s'agit de rythmes associés à la musique instrumentale qui accompagne la danse. Ces rythmes se caractérisent par l'agglomération de deux unités de durée (brève-longue) au lieu d'une seule, et leur relation mathématique est irrationnelle : chaque unité, plutôt que de valoir la moitié ou le double de l'autre, lui est en relation de $2 / 3$ ou de $3 / 2$ (Brailoiu 1973). En Espagne, la présence de tels rythmes a été signalée dans la musique traditionnelle basque (Brailoiu, ibid.), catalane (Aiats 1990) et valencienne (Ortí 1988; Torrent i Centelles 1990; Pelinski 1991).

Bien qu'il ne soit pas possible de déterminer avec précision l'ancienneté de la présence de ces rythmes dans la Danse guerrière, je puis affirmer, en me fondant sur les considérations précédentes, que ces rythmes font partie d'une strate de durée séculière, laquelle, aujourd'hui, à cause de son interaction avec l'omniprésente musique commerciale, est en voie de disparition.

4.4. Je dois à une communication personnelle de Monsieur Vicent Serra i Fortuño la perception de la similitude existant entre les figures chorégraphiques 
de la première partie du Torn $\left(\mathrm{n}^{\circ} 13\right)$ et la description de la Dança de espadas («Danse des épées ») donnée par Sebastián de Covarrubias y Orozco dans son renommé Tesoro de la Lengua Castellana o Española (1611) :

Danse des épées. Cette danse est en usage au royaume de Tolède, et elle est dansée en chemise et grègues d'étoffe, avec des ornements sur la tête, et ils portent des épées blanches et font avec elles de grands tours et une figure qu'ils appellent l'« égorgement » parce qu'ils entourent le cou de celui qui les guide [le « chef de danse »] avec les épées et quand il semble qu'ils vont lui couper la tête, il s'échappe.

Il est possible, donc, que cette figure la Danse (l' " égorgement ») remonte à une tradition déjà connue au début du XVII ${ }^{\mathrm{e}}$ siècle. En revanche, la Jota de la seconde partie du Torn ne peut être plus ancienne que la danse homonyme qui, selon les témoignages historiques, apparaît en Espagne au milieu du XVIII siècle (Pujol et Amades 1936).

4.5. Le Mambrú ( $\left.\mathrm{n}^{\circ} 12\right)$ qui, comme l'indique Vicent Serra i Fortuño (1988), se retrouve aussi dans les danses de Peñiscola et dans le Ball pla ${ }^{14}$ de Benassal, est une mélodie populaire du début du $\mathrm{XIX}^{\mathrm{e}}$ siècle et il ne serait pas impensable qu'on l'ait ajoutée à la Danse à cette époque. Selon une communication personnelle de María Antonia Virgili Blanquet, Luis Villalba, dans un article publié dans Ciudad de Dios (Escorial, 1909), mentionne la diffusion du Mambrú en relation avec les guerres napoléonniennes.

Cette brève analyse suffira à montrer que les mélodies de la Danse guerrière correspondent à différents stades historiques et stylistiques. À l'exemple des pratiques communes de la musique traditionnelle de plusieurs pays, la Danse guerrière semble être le fruit d'un processus de bricolage dans lequel les paysans ajoutent, déplacent et rejettent des pièces, sans que la Danse elle-même, comme expression culturelle d'une communauté, perde son identité et son pouvoir de ralliement.

Ces changements n'empêchent pas que, dans l'intention d'établir des liens génético-historiques, on puisse vaguement inscrire la Danse guerrière dans la tradition des danses d'épées (ou de bâtons) attestées à Valence à partir de 1439, lorsqu'une danse d'épées fut exécutée par la Corporation des forgerons, ou, plus anciennement encore, dans l'antique tradition des danses pyrrhiques grecques (Pujol et Amades 1936).

14 « Danse simple ». 


\section{Conclusion}

Les réflexions précédentes me semblent offrir suffisamment d'évidences pour permettre d'avancer que la créativité de la mémoire orale peut compenser l'absence de sources historiques écrites, en nous livrant des connaissances importantes sur la structure, la conceptualisation et le contexte socio-culturel d'une pratique artistique traditionnelle. Le Don Quichottisme, c'est-à-dire « le désir obsessif de trouver le passé derrière le présent » (Lévi-Strauss 1988), peut être décevant, non seulement parce qu'il nous révèle la pauvreté des origines (Nietzsche 1980 : 47), mais aussi parce qu'il nous cache éventuellement la perspective d'une tradition qui, tenant compte des mots, des images, des geste, des rituels et de la fête des oubliés de l'histoire, peut élargir l'horizon de l'histoire écrite (Le Goff 1988 : 170, 221). L'histoire orale du présent peut devenir l'histoire écrite du futur.

D'un autre côté, il ne faut pas rejeter les documents historiques faux : je crois en effet qu'en les interprétant au second degré, nous pouvons vérifier certaines constantes culturelles qui illustrent des aspects de la vie quotidienne paysanne. La construction de ces discours symboliques autour de la Danse, aussi bien que l'expérience actuelle et vécue de sa pratique, constituent la mémoire collective sur laquelle repose le sentiment d'identité d'une communauté. Narrations écrites et mémoires orales, histoire et anthropologie se retrouvent dans la musicologie.

Il me semble donc raisonnable que de penser que l'histoire de la Danse guerrière est condensée dans sa pratique actuelle. Une pratique conçue comme un texte en état de production continue, et non comme une reproduction de quelque chose de figé, transmis à travers les siècles.

J'aimerais finir ces réflexions en reformulant le problème dans les termes suivants : Aristote a défini l'être comme « ce qui était », comme permanence du passé. Quant à moi, je préfère concevoir la pratique de la Danse guerrière en termes héguéliens : non comme ce qu'elle a été, mais comme ce que elle est, en tant que son état actuel contient et dépasse en même temps son origine. Cette conception des origines d'une pratique culturelle, libérée du poids obsessif de l'histoire, nous permet de penser que la Danse guerrière fut ce qu'elle est aujourd'hui et qu'elle sera, peut-être, demain : une expression de la culture toujours ancienne et toujours renouvelée d'un village du Maestrazgo.

\section{RÉFÉRENCES}

AIATS, J.

1990 : El ritme g. s. 1212 : un cas notable de giusto sillabic en les cançons baladístiques de la comarca d'Osona (Ms.). 
AMADES, J.

1955 : «Las Danzas de espadas y de palos en Cataluña, Baleares y Valencia », Anuario Musical, X, 163-187.

BAUMANN, M.P.

1976 : Musikfolklore und Musikfolklorismus. Winterthur : Amadeus.

BOUCHÉ PERIS, $\mathrm{H}$.

1982 : «Fuego, demonios y santos. Un estudio de antropología castellonense ", Estudios Castellonenses, 1, 185-229.

BRAUDEL, F.

1968 : La Historia y las ciencias sociales. Madrid : Alianza.

BRAILOIU, C.

1973 : «Le rythme aksak » (1951), Problèmes d'ethnomusicologie. Genève : Minkoff.

CARRERAS ALVAR, A.M.

1980 : « Las danzas guerreras en Todolella y Peñiscola », Narria, 17, Universidad Autónoma de Madrid, 20-24.

CERTEAU, M. de

1974 : « L'opération historique »,Faire de l' Histoire,I(J.Le Goff et P.Nora,édit.). Paris : Gallimard.

COVARRUBIAS DE OROZCO, S.

1611 : Tesoro de la Lengua Castellana o Española.

DETIENNE, $M$.

1986 : « Le Mythe », Faire de l' Histoire, III (J. Le Goff et P. Nora, édit.). Paris : Gallimard.

GARCÍA MATOS, M.

1959-61 : "Viejas canciones y melodías en la música instrumental popular de las danzas procesionales practicadas aún en España », Miscelánea en homenaje a Monseñor Higinio Anglés, I, Barcelone.

GEORGIADES, T.G.

1949 : Der Griechische Rhythmus, Musik, Reigen, Vers und Sprache, Hambourg.

HOBSBAWN, J.E.

1972 : «The social function of the past : some questions », Past and Present, 55, 3-17.

HOMENAJE a MOSSÉN MILÍAN, 2

1987 : Diputación de Castellón. 
JOUTARD, P.

1977 : La Légende des Camisards : une sensibilité au passé. Paris : Gallimard.

LE GOFF, J.

1988 : Histoire et mémoire. Paris : Gallimard.

LÉVI-STRAUSS, C.

1988 : De près et de loin. Paris : Plon.

MARTÍ I PÉREZ, J.

1990 : «El folklorismo. Análisis de una tradición 'prêt-à-porter' », Anuario Musical, 45 .

MILIÁN BOIX, MOSSÉN M.

1950 : «La Dança Todolellana. Fué transmitida cuidadosamente de padres a hijos ", Mediterráneo, 24-2-1950, 2.

NIETZSCHE, F.

1980 : Aurore. Paris : Gallimard.

NORA, P.

1978 : «Présent », in J. Le Goff, La Nouvelle Histoire. Paris : Retz, 467-472.

1984 : Les Lieux de mémoire, I : La République. Paris : Gallimard.

OLMOS, R.

1951 : Cuadernos de música folklórica valenciana, 9 : Canciones y Danzas de Morella, Forcall, Todolella, Castell de Cabres.

ORTI, A.

1988 : Notes du disque Morella.Tocs tradicionals de dolçaina. Valence : Conselleria de Cultura, Educació i Ciencia. Fonoteca de Materials. Tallers de Música Popular. VII.

PALAU, M.

1951 : Cuadernos de música folklórica valenciana, II, 7-8: Canciones y danzas de Morella y Peñiscola.

PELINSKI, R.

1991 : Notes du disque Danses $i$ Cançons de La Todolella. Tallers de Música Popular de la Conselleria de Cultura, Educació i Ciencia de la Generalitat Valenciana.

PUJOL, F. et AMADES, J.

1936 : Diccionari de la dansa, dels entremesos $i$ dels instruments de musica $i$ sonadors, I : Dansa, del Cançoncer popular de Catalunya, Barcelone.

PUERTO MEZQUITA, G.

1956 : Danzas procesionales de Morella y del Maestrazgo. 
QUEROL GALVADÁ, M.

1956: «El villano de la época de Cervantes y su supervivencia en el folklore contemporáneo », Anuario Musical, XI.

SERRA I FORTUÑO, V.

1988 : «Notes varies a les Danses Guerreres ». Programa de los festejos que La Todolella dedica a su patrón San Bartolomé, apóstol y mártir, bajo los auspicios del Magnífico Ayuntamiento.

TORRENT I CENTELLES, $\mathrm{V}$.

1990 : Ritmes amalgamats en la tradició valenciana (Ms.).

WIDDES, $\mathbf{R}$.

1989 : «Introduction ", Ethnomusicology and the historical dimension (M.L. Philipp, éd.). Ludwigsburg : Philipp. 